\title{
Comparison of Pregnancy Outcomes of High-Quality D5- and D6-Blastocyst Transfer in Hormone-Replacement Frozen-Thawed Cycles
}

\author{
Weijie Xing, Liuhong Cai, Li Sun, Jianping Ou* \\ Center for Reproductive Medicine, The Third Affiliated Hospital of Sun Yat-sen University, Guangzhou, China \\ Email: *oujianping1968@qq.com
}

How to cite this paper: Xing, W.J., Cai, L.H., Sun, L. and Ou, J.P. (2017) Comparison of Pregnancy Outcomes of High-Quality D5- and D6-Blastocyst Transfer in HormoneReplacement Frozen-Thawed Cycles. International Journal of Clinical Medicine, 8, 565-571. https://doi.org/10.4236/ijcm.2017.811053

Received: September 27, 2017

Accepted: November 12, 2017

Published: November 15, 2017

Copyright (๑) 2017 by authors and Scientific Research Publishing Inc. This work is licensed under the Creative Commons Attribution International License (CC BY 4.0).

http://creativecommons.org/licenses/by/4.0/

\begin{abstract}
This study aimed to assess pregnancy outcomes after high-quality D5- and D6-blastocyst transfer in frozen cycles of in vitro fertilization and embryo transfer and to further evaluate whether there was a difference in blastocyst development potentials with different developmental speeds and in pregnancy outcomes. A retrospective analysis was conducted to analyze 247 frozen cycles in our center from September 2015 to July 2017, which were divided into two groups: a D5-FET group with 193 cycles of D5-blastocyst transfer, and a D6-FET group with 54 cycles of D6-blastocyst transfer. Hormone replacement method was utilized to prepare frozen-cycle endometria. Pregnancy outcomes were analyzed and compared between these two groups. The mean ages of the two groups were $31.45 \pm 4.43$ years and $31.98 \pm 4.84$ years, respectively, with no statistically significant differences $(P>0.05)$. The difference in the endometrial thickness during transfer was also not statistically significant. The implantation rate in the D5-FET group was $60.13 \%$, significantly higher than that in the D6-FET group $(31.58 \%, P<0.05)$. The clinical pregnancy rate in the D5-FET group was $68.91 \%$, significantly higher than that in the D6-FET group $(40.74 \%, P<$ $0.05)$. No statistically significant differences were found in the abortion rate and ectopic pregnancy rate between the two groups. The implantation, biochemical pregnancy, and clinical pregnancy rates of the blastocyst D5 were all superior to those of the blastocyst D6. In clinics, therefore, D5-blastocyst transfer could be prioritized for embryo transfer.
\end{abstract}

\section{Keywords}

Frozen Cycle, Hormone Replacement Therapy, Blastocyst Transfer 


\section{Introduction}

With a constant improvement in the embryo culture system, blastocyst culture has been widely used in clinics. Blastocyst culture is of more benefit to selecting an embryo with a development potential. Blastocyst transfer produces a better synchronization between embryo development and the endometrium. In addition, because of reduced movement of the endometrium during blastocyst transfer, blastocyst transfer has a better pregnancy outcome than embryo transfer during the cleavage period. Moreover, single blastocyst transfer has a higher pregnancy rate and significantly reduces multiple-gestation pregnancy rate.

During the process of in vitro embryo culture, embryo development speeds are not consistent. In general, a blastocyst is formed on day 5 (D5) or day 6 (D6) after fertilization. With respect to the difference in blastocyst development potential during different development periods, a consistent conclusion has not yet been achieved. Some studies suggest that both the clinical pregnancy rate and implantation rate of D5-blastocyst transfer were higher than those of D6 during a fresh transfer cycle [1], whereas El-Toukhy et al. reported no significant differences in these two rates after frozen-thawed D5- or D6-blastocyst transfer during a freeze-thaw cycle of blastocyst transfer [2] [3] [4]. Mesut et al. [5], however, reported that the clinical pregnancy, implantation, and birth rates of frozen-thawed D5-blastocyst transfer were significantly higher than those of D6. Therefore, this study aimed to evaluate pregnancy outcomes of high-quality D5and D6-blastocyst transfer based on a retrospective analysis of frozen cycles of in vitro fertilization and embryo transfer and to further assess whether a difference is found in blastocyst development potentials with different development speeds and in pregnancy outcomes, thereby providing a guide for embryo selection on the day of thawing.

\section{Materials and Methods}

A retrospective analysis of freeze-thaw cycles was conducted in our center from September 2015 to July 2017. This retrospective study was approved by the Third Affiliated Hospital of Sun Yat-sen University Medicine Ethics Committee. Patient records and information were anonymized and de-identified prior to analysis. The inclusive criteria were: 1) the cycles wherein D5- or D6-blastocyst transfer was performed; 2) hormone replacement cycle; and 3) the embryos transferred were high-quality blastocysts. The exclusive criteria were: 1) natural cycles or ovarian stimulation cycles; and 2) the embryos transferred were not high-quality blastocysts. A total of 247 frozen cycles were included and divided into two groups: the D5-FET group with 193 cycles of D5-blastocyst transfer and the D6-FET group with 54 cycles of D6-blastocyst transfer.

The protocol of controlled ovarian hyperstimulation for patients in an oocyte retrieval period was based on the routine practice in our center. After oocyte retrieval, patients underwent either natural fertilization or intracytoplasmic sperm injection (ICSI) fertilization based on their conditions. Fertilization was observed 
$24 \mathrm{~h}$ after oocyte retrieval, and blastomere-stage embryos were observed $72 \mathrm{~h}$ after oocyte retrieval. Transfer was determined based on embryonic scores. All remnant embryos after transfer were used for blastocyst culture after the patients provided an informed consent. Blastocyst formation and configuration on day 5 or 6 after fertilization were observed, respectively. Blastocysts were scored based on the Gardner score system [5]. First, blastocysts were staged based on the dilation degree of the cyst cavity. The stage wherein the cyst cavity has a dilation of $<50 \%$ of the embryo length was called Stage 1 ; the stage wherein the cyst cavity has a dilation of $>50 \%$ of the embryo length was called Stage 2 ; the stage wherein the cyst cavity was occupied by the entire embryo was called Stage 3; the stage wherein there was blastocyst growth with a thinning of the zona pellucida was called Stage 4; the stage wherein the blastocyst began to incubate was called Stage 5 ; the stage wherein the blastocyst was completely incubated was called Stage 6. The blastocysts in Stages 4 - 6 were further evaluated. The inner cell mass (ICM) scoring was as follows: A, a large number of cells with a dense arrangement; $B$, a small number of cells with a loose arrangement; $C$, a few numbers of cells. The trophectoderm (TE) evaluation was as follows: A, a large number of cells surrounding the entire cyst cavity with a dense arrangement; $B$, a small number of cells with a loose arrangement; $\mathrm{C}$, a few numbers of cells. Because a previous observation indicated that the early and incubated blastocysts had an unsatisfied thaw rate after being frozen, the blastocysts in Stage 4 in our center were frozen and stored. The blastocysts with grade A or B ICM were selected to be frozen, whereas those with a grade $\mathrm{C}$ quality were abandoned. A high-quality blastocyst was defined as the blastocyst in Stages $4-6$ with a grade A or B quality [5].

Our center used vitrification freezing to freeze the embryos, which was based on the previously described [6]. The blastocysts were subjected to artificial shrinkage before being frozen, and then allowed to equilibrate for $2 \mathrm{~min}$ in a pre-equilibrated solution containing 7.5\% (v/v) dimethylsulfoxide (DMSO) and 7.5\% (v/v) ethylene glycol (EG), followed by transfer to a vitrification freezing solution-15\% (v/v) $\mathrm{DMSO}+15 \%(\mathrm{v} / \mathrm{v}) \mathrm{EG}+0.65 \mathrm{~mol} / \mathrm{l}$ sucrose $+10 \mathrm{mg} / \mathrm{ml}$ Ficoll-where the blastocysts were allowed to equilibrate for $30 \mathrm{~s}$. Subsequently, the blastocysts were transferred to a Cryoloop, which was then preserved in liquid nitrogen. Blastocyst thaw was conducted on the same day with embryo transfer. Before the blastocysts were thawed, a series of blastocyst thaw solutions were prepared by making a $0.33 \mathrm{~mol} / \mathrm{l}$ sucrose solution, a $0.2 \mathrm{~mol} / 1$ sucrose solution, and a basic culture solution (G-MOPS Plus, Vitrolife, Sweden) in an embryo culture dish. The Cryoloop containing the blastocysts was gathered from the liquid nitrogen and allowed to stay in the air for $5 \mathrm{~s}$ before it was directly soaked in the abovementioned thaw solutions for $2 \mathrm{~min}, 3 \mathrm{~min}$, and $5 \mathrm{~min}$, respectively. The blastocysts were transferred to a pre-made blastocyst culture solution (G2, Vitrolife, Sweden) and cultured for $2 \mathrm{~h}$ in an incubator filled with $6 \% \mathrm{CO}$ and $5 \% \mathrm{O}_{2}$ at $37^{\circ} \mathrm{C}$. Before blastocyst transfer, blastocyst survival was evaluated, with re-dilating of the blastocyst cavity as an indicator of blastocyst survival. 
The endometria were prepared with hormone replacement therapy: a routine B-ultrasound examination was arranged on days 2 to 5 during menstruation, and estradiol valerate (Progynova, Schering, Germany) was started to be orally administered at a dose of $2 \mathrm{mg}$ bid, and the dose was altered to $3 \mathrm{mg}$ bid 5 days later, with oral administration of the drug being continued for another 5 days. After 10-day oral administration, B-ultrasound was re-examined. When the thickness of endometrium was $>8 \mathrm{~mm}$, progesterone was intramuscularly injected at a dose of $40 \mathrm{mg} /$ day. On day 6 of the progesterone administration, D5- or D6-blastocyst transfer was performed.

On day 12 after embryo transfer, a positive result of a urine pregnancy test indicated biochemical pregnancy, and blood hCG levels were also tested at the same time. If a gestational sac (GS) was observed by ultrasound examination after 3 weeks, it was considered a clinical pregnancy. If the GS was not implanted in the uterus as the B-ultrasound indicated, it was considered an ectopic pregnancy. Pregnancy loss before 28 weeks was considered abortion.

SPSS13.0 statistic software was used for analysis. Measurement data were expressed as mean \pm standard deviation. Comparison between the two groups was analyzed with t-test. Enumeration data were analyzed with chi-square test. $P<$ 0.05 was set with a statistically significant difference.

\section{Results}

A total of 247 frozen cycles were analyzed, including 193 cycles in the D5-FET group and 54 cycles in the D6-FET group. The mean ages of the two groups were $31.45 \pm 4.43$ years and $31.98 \vee 4.84$ years, respectively, with no statistically significant differences $(P>0.05)$. As showed in Table 1 , the difference in the thickness of endometrium during transfer was also not statistically significant $(9.77 \pm 1.06 \mathrm{~mm}$ vs. $9.52 \pm 1.50 \mathrm{~mm}, P>0.05)$. The number of transferred embryos in the D6-FET group was $1.76 \pm 0.47$, which was significantly more than that in another group $(1.61 \pm 0.49, P<0.05)$. However, the number of implanted

Table 1. The clinical materials and outcomes of frozen ET.

\begin{tabular}{cccc}
\hline Variables & $\begin{array}{c}\mathrm{D} 5 \\
\mathrm{~N}=193)\end{array}$ & $\begin{array}{c}\mathrm{D} 6 \\
(\mathrm{~N}=54)\end{array}$ & $\begin{array}{c}P \text { value } \\
\text { Age }\end{array}$ \\
$\begin{array}{ccc}\text { Ne } \\
\text { No. of transferred embryos }\end{array}$ & $1.61 \pm 0.49$ & $31.98 \pm 4.84$ & $\mathrm{NS}$ \\
No. of implanted embryos & $0.96 \pm 0.77$ & $1.76 \pm 0.47$ & $<0.05$ \\
Thickness of endometrium & $9.77 \pm 1.06$ & $0.56 \pm 0.74$ & $<0.05$ \\
Implantation rate & $60.13 \%(187 / 311)$ & $31.58 \%(30 / 95)$ & $\mathrm{NS}$ \\
Biochemical pregnancy rate & $74.61 \%(144 / 193)$ & $48.15 \%(26 / 54)$ & $<0.05$ \\
Clinical pregnancy rate & $68.91 \%(133 / 193)$ & $40.74 \%(22 / 54)$ & $<0.05$ \\
Miscarriage rate & $11.28 \%(15 / 133)$ & $9.09 \%(2 / 22)$ & $\mathrm{NS}$ \\
Ectopic pregnancy rate & $0.75 \%(1 / 133)$ & $0 \%(0 / 22)$ & $\mathrm{NS}$ \\
\hline
\end{tabular}

Note: "NS" means "no significant, $P>0.05$ ". 
embryos in the D6-FET group was dramatically less than that in the D5-FET group $(0.56 \pm 0.74$ vs. $0.96 \pm 0.77, P<0.05)$. The implantation rate in the D5-FET group was $60.13 \%$, which was significantly higher than that in the D6-FET group (31.58\%, $P<0.05)$. The biochemical pregnancy rate in the D5-FET group was significantly higher than that in the D6-FET group $(74.61 \%$ vs. $48.15 \%, P<$ 0.05). The clinical pregnancy rate in the D5-FET group was $68.91 \%$, which was significantly higher than that in the D6-FET group $(40.74 \%, P<0.05)$. No statistically significant differences were found in the abortion and ectopic pregnancy rates between the two groups $(11.28 \%$ vs. $9.09 \%$; $0.75 \%$ vs. $0 \% ; P>0.05)$.

\section{Discussion}

This study retrospectively analyzed the clinical data of pregnancy after D5- and D6-blastocyst transfer in freeze-thaw cycles, and the results were that the implantation, biochemical pregnancy, and clinical pregnancy rates of the D5 blastocyst were significantly higher than those of the D6 blastocyst, which are consistent with the results of previous studies [3].

An issue exists on what caused the above results-is it because freeze-thaw may cause different effects on blastocysts D5 and D6, or because different blastocyst development stages may cause different development potentials? Kenichiro et al. frozen and thawed D5 and D6 blastocysts and found that the survival rate of these two types of blastocysts were $99.5 \%$ and $97.9 \%$, respectively, with no statistically significant differences [4]. In this study, we also found that no difference was found in the thaw rate of D5 and D6 blastocysts. The results discussed above indicated that vitrification freezing for blastocysts formed in different stages had no effect on the survival rate of post-thawed embryos.

To date, an issue that in which stages the D5 and D6 blastocysts can have a better development potential has not yet achieved a consistent conclusion. Kenichiro et al. found that no statistically significant differences were observed in the implantation and pregnancy rates between D5 and D6 blastocysts [4]. Some studies, however, indicated that the implantation rate of D5 blastocyst after being frozen and thawed was significantly higher than that in the D6-blastocyst group, whereas the clinical pregnancy rate of the D5 blastocyst was slightly higher than that in the D6 group, but with no statistically significant difference [7]. A recent meta-analysis included 15 articles of frozen-thawed blastocyst transfer, including 2512 freeze-thaw cycles of blastocyst transfer [3]. In that meta-analysis study, the clinical pregnancy, ongoing pregnancy, and live birth rates of frozen-thawed blastocysts in the D5 group were found to be significantly higher than those in the D6 group. However, after subgroup comparison of clinical pregnancy outcomes of frozen-thawed D5 and D6 blastocysts developed in the same stage, no statistically significant differences were observed.

All blastocysts selected in this study were high-quality blastocysts, and the results showed that the implantation rate in the D5-FET group was $6013 \%$, significantly higher than that in the D6-FET group $(31.58 \%, P<0.05)$. The biochemi- 
cal pregnancy rate in the D5-FET group was significantly higher than that in the D6-FET group $(74.61 \%$ vs. $48.15 \%, P<0.05)$. The clinical pregnancy rate in the D5-FET group was $68.91 \%$, significantly higher than that in the D6-FET group (40.74\%, $P<0.05)$. No statistically significant differences were found in the abortion and ectopic pregnancy rates between the two groups (11.28\% vs. 9.09\%; $0.75 \%$ vs. $0 \% ; P>0.05)$. The abovementioned results were similar to those of the study by Kovalevsky et al., who compared blastocysts in the three development stages D5, D6, and D7 and found that the implantation, clinical pregnancy, and ongoing pregnancy rates of the D5 blastocyst were significantly higher than those of blastocysts in the D6 and D7 groups [6]. Similarly, the results of the study by Liebermann et al. also suggested that the pregnancy outcome of D5 was better than that of the D6 blastocyst [8].

The reasons why the pregnancy outcome of the D5 blastocyst was better than that of D6 were primarily: 1) some researchers believed that the embryo development speed could affect the outcomes of frozen-thawed blastocyst transfer, and the delayed formation of blastocysts meant a formation of poor-quality embryos; 2) some studies conducted a trophoblastic biopsy for chromosome analysis and found that the incidence of aneuploid occurring in blastocysts with a delayed development was much higher [9] [10].

There were some limitations as follows: 1) it is a retrospective study; 2) the sample size was small. So, the results should be confirmed by further, adequately sized studies, or meta-analyzed with similar studies.

\section{Conclusion}

The implantation, biochemical pregnancy, and clinical pregnancy rates of the D5 blastocysts were all superior to those of the D6 blastocysts. In clinics, therefore, D5 blastocyst transfer could be prioritized for embryo transfer.

\section{References}

[1] Maheshwari, A., Griffiths, S. and Bhattacharya, S. (2011) Global Variations in the Uptake of Single Embryo Transfer. Human Reproduction Update, 17, 107-120. https://doi.org/10.1093/humupd/dmq028

[2] El-Toukhy, T., Wharf, E., Walavalkar, R., Singh, A., Bolton, V., Khalaf, Y. and Braude, P. (2011) Delayed Blastocyst Development Does Not Influence the Outcome of Frozen-Thawed Transfer Cycles. International Journal of Obstetrics \& Gynaecology, 118, 1551-1556. https://doi.org/10.1111/j.1471-0528.2011.03101.x

[3] Sunkara, S.K., Siozos, A., Bolton, V.N., Khalaf, Y., Braude, P.R. and El-Toukhy, T. (2010) The Influence of Delayed Blastocyst Formation on the Outcome of Frozen-Thawed Blastocyst Transfer: A Systematic Review and Meta-Analysis. Human Reproduction, 25, 1906-1915. https://doi.org/10.1093/humrep/deq143

[4] Hiraoka, K., Hiraoka, K., Miyazaki, M., Fukunaga, E., Horiuchi, T., Kusuda, T., Okano, S., Kinutani, M. and Kinutani, K. (2009) Perinatal Outcomes Following Transfer of Human Blastocysts Vitrified at Day 5, 6 and 7. Journal of Experimental \& Clinical Assisted Reproduction, 6, 4.

[5] Mesut, N., Ciray, H.N., Mesut, A., Aksoy, T. and Bahceci, M. (2011) Cryopreserva- 
tion of Blastocysts Is the Most Feasible Strategy in Good Responder Patients. Fertility and Sterility, 96, 1121-1125. https://doi.org/10.1016/j.fertnstert.2011.08.012

[6] Kovalevsky, G., Carney, S.M., Morrison, L.S., Boylan, C.F., Neithardt, A.B. and Feinberg, R.F. (2013) Should Embryos Developing to Blastocysts on Day $7 \mathrm{Be}$ Cryopreserved and Transferred: An Analysis of Pregnancy and Implantation Rates. Fertility and Sterility, 100, 1008-1012.

https://doi.org/10.1016/j.fertnstert.2013.06.021

[7] Levens, E.D., Whitcomb, B.W., Hennessy, S., James, A.N., Yauger, B.J. and Larsen, F.W. (2008) Blastocyst Development Rate Impacts Outcome in Cryopreserved Blastocyst Transfer Cycles. Fertility and Sterility, 90, 2138-2143.

https://doi.org/10.1016/j.fertnstert.2007.10.029

[8] Liebermann, J. (2009) Vitrification of Human Blastocysts: An Update. Reproductive Biomedicine Online, 19, 4328. https://doi.org/10.1016/S1472-6483(10)61073-5

[9] Majumdar, G., Majumdar, A., Verma, I.C. and Upadhyaya, K.C. (2017) Relationship between Morphology, Euploidy and Implantation Potential of Cleavage and Blastocyst Stage Embryos. Journal of Human Reproduction Science, 10, 142-150. https://doi.org/10.4103/jhrs.JHRS_98_17

[10] Alfarawati, S., Fragouli, E., Colls, P., Stevens, J., Gutierrez-Mateo, C., Schoolcraft, W.B., Katz-Jaffe, M.G. and Wells, D. (2011) The Relationship between Blastocyst Morphology, Chromosomal Abnormality, and Embryo Gender. Fertility and Sterility, 95, 520-524. https://doi.org/10.4103/jhrs.JHRS_98_17 